\title{
Medical Image of the Week: Hemophagocytic Lymphohistiocytosis (HLH)
}

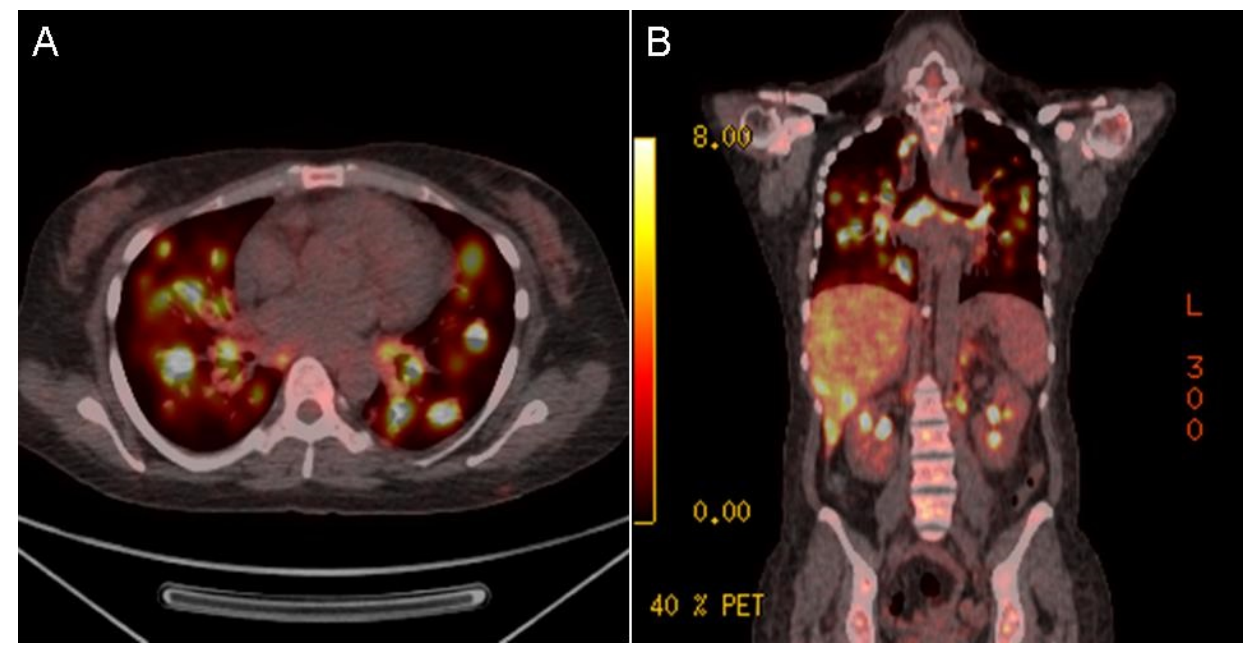

Figure 1. Axial (Panel A) and coronal (Panel B) PET-CT Scan Images showing numerous metabolically active pulmonary nodules and mediastinal lymph nodes.

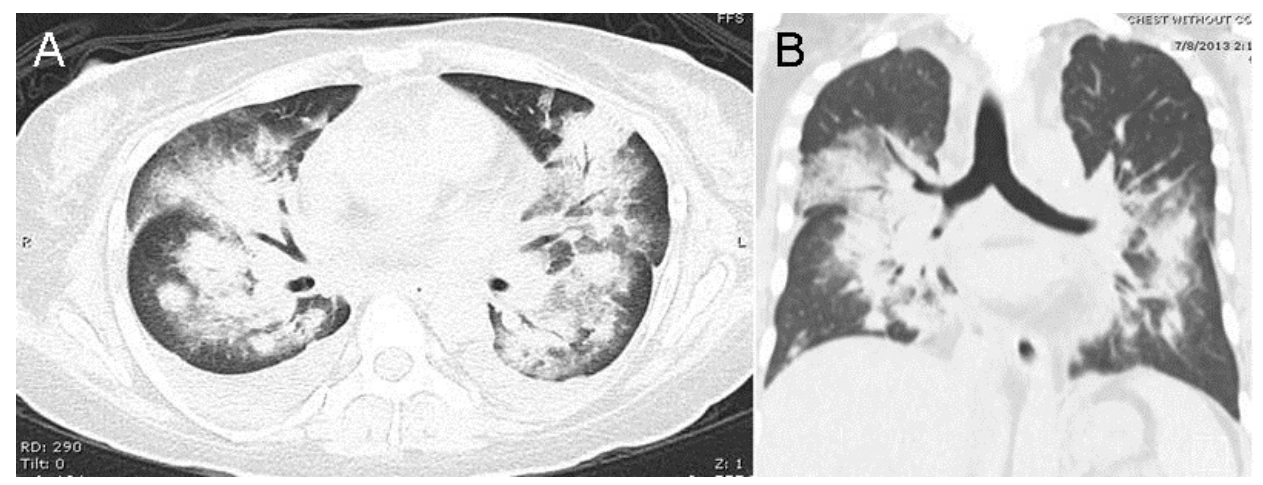

Figure 2. Axial (Panel A) and Sagittal (Panel B) images of the chest CT showing consolidative pulmonary nodules with surrounding ground glass halo and mediastinal lymphadenopathy.

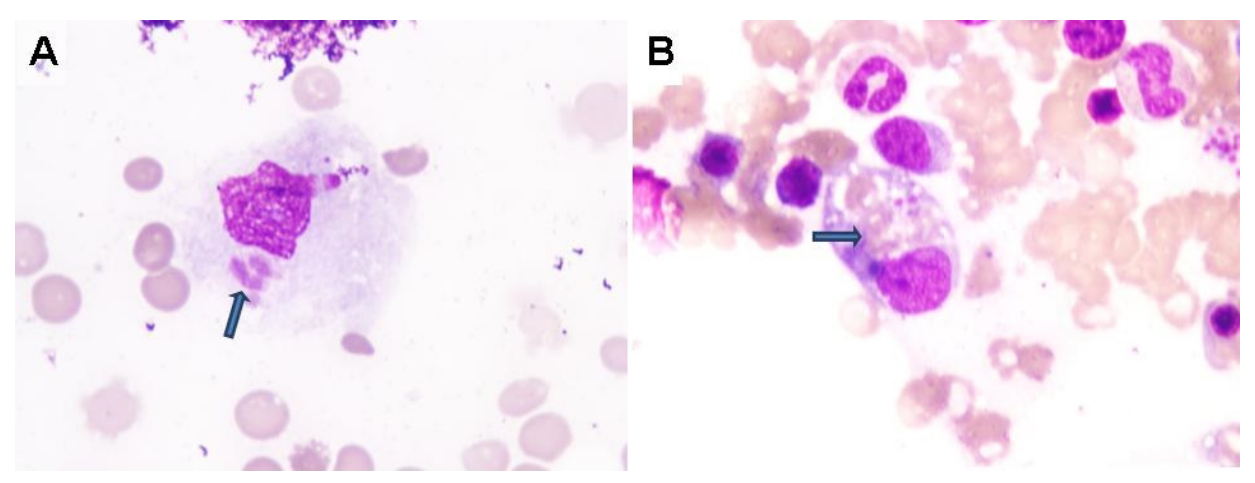

Figure 3. Bone Marrow Biopsy (x1000). Panel A: macrophage engulfing a neutrophil. Panel B: macrophage engulfing erythrocyte debris. 


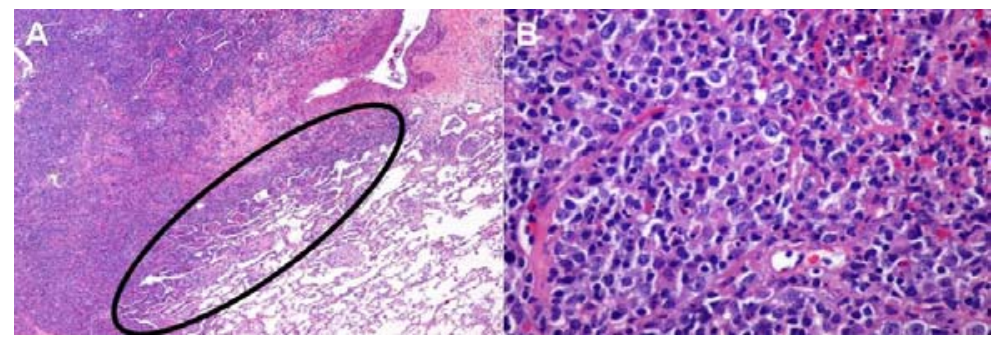

Figure 4. Panel A: Lower power view of the lung biopsy (H\&E stain) showing the interface between the neoplastic lymphocytic infiltrate and benign, normal lung. Panel B:

high power view showing the neoplastic B-cell lymphoma with sheets of large lymphocytes.

A 41-year-old African American woman with a history of diffuse large B cell lymphoma in remission was admitted to the hospital with severe dyspnea and abdominal pain. Recent imaging revealed extensive pulmonary and liver nodules with significant mediastinal lymphadenopathy (Figures 1 and 2). She had an extensive outpatient evaluation of these abnormalities including multiple percutaneous and endoscopic biopsies which were nondiagnostic. She deteriorated clinically and a ferritin level was elevated at $36,284 \mathrm{ng} / \mathrm{mL}$. Due to the markedly elevated ferritin, a bone marrow biopsy was performed and was normocellular with trilineage hematopoiesis and erythrophagocytosis consistent with hemophagocytic lymphohistiocytosis (HLH, Figure 3). A VATS guided lung biopsy was performed revealing recurrence of the diffuse large B cell lymphoma (Figure 4). She started chemotherapy with the E-SHAP (etoposide, methylprednisolone, cytarabine, cisplatin), however, became severely pancytopenic and developed acute respiratory failure, shock and multi-organ failure. She died despite aggressive care in the intensive care unit. Patients with HLH often present with sepsis like symptoms and multiorgan failure. Measurement of serum ferritin level is a critical test in suggesting the diagnosis once infection is excluded. Early recognition and prompt treatment is essential to preventing fatal outcomes.

Tauseef Afaq Siddiqi, MD; Carlos Tafich Rios, MD²; Carlos L Cantu, MD³; James Knepler, MD ${ }^{1}$; Linda Snyder, MD ${ }^{1}$

${ }^{1}$ Division of Pulmonary, Allergy, Critical Care and Sleep Medicine,

2 Department of Medicine,

${ }^{3}$ Department of Pathology, The University of Arizona, Tucson, AZ 85724, USA.

\section{References}

1. Raschke RA, Garcia-Orr R. Hemophagocytic lymphohistiocytosis: a potentially underrecognized association with systemic inflammatory response syndrome, severe sepsis, and septic shock in adults. Chest. 2011;140(4):933-8. [CrossRef] [PubMed]

2. Okabe T, Shah G, Mendoza V, Hirani A, Baram M, Marik P. What intensivists need to know about hemophagocytic syndrome: an underrecognized cause of death in adult intensive care units. J Intensive Care Med. 2012;27(1):58-64. [CrossRef] [PubMed] 